\title{
Impact of hematologic toxicities during concurrent chemoradiation for cervical cancer
}

\author{
Feiya Shi, B.A. ${ }^{1,{ }^{*}}$, Alison K. Yoder, MD, MPH ${ }^{2,}$, , Claire Mach, MD ${ }^{3}$, Shraddha Dalwadi, MD, MBA ${ }^{4}$, \\ Matthew L Anderson, MD, PhD ${ }^{5}$, Tracilyn R Hall, MD³, Michelle S Ludwig, MD, PhD ${ }^{4}$ \\ Department of Radiation Oncology, ${ }^{1}$ Baylor College of Medicine, ${ }^{2}$ University of Texas Health Science Center at Houston McGovern School of Medicine, \\ ${ }^{3}$ Department of Obstetrics and Gynecology, ${ }^{4}$ Department of Radiation Oncology, Baylor College of Medicine, Houston, TX; ${ }^{5}$ Division of Gynecologic \\ Oncology, Morsani College of Medicine, University of South Florida/Moffitt Cancer Center, Tempa, FL, USA
}

\section{Objective}

To evaluate the prognostic significance of hematological toxicities during cervical cancer treatment.

Methods

Patients treated for cervical carcinoma with definitive chemoradiation were identified. Toxicities were assessed during weeks 1 to 6 of concurrent external beam radiation and chemotherapy. Outcomes were analyzed using Cox regression analysis.

\section{Results}

One hundred twenty-one patients with Federation of Gynecology and Obstetrics stage I-III disease were eligible for analysis. Median age at diagnosis was 45 years (interquartile range, 40-52) with median follow-up time of 34 months (95\% confidence interval, 30.8-37.2). All patients experienced some grade of hematologic toxicity. The most common grade $3+$ toxicities were low absolute lymphocyte count $(n=115,95 \%)$, low white blood cell count $(n=21,17 \%)$, and anemia $(n=11,9 \%)$. The most common grade 4 toxicity was lymphopenia, experienced by $36 \%$ of patients $(n=44)$. Grade 4 lymphopenia was associated with reduced overall survival (hazard ratio [HR], 4.5; $P=0.005$ ), progressionfree survival (HR, 3.4; $P=0.001)$, and local control (HR, 4.1; $P=0.047)$. Anemia grade 3, 4 was also associated with reduced overall survival $(H R, 4.1 ; P=0.014)$. After controlling for disease and treatment variables, grade 4 lymphopenia remained significantly associated with reduced overall survival $(H R, 9.85 ; P=0.007)$. The association with grade 4 lymphopenia only remained significant in women of Hispanic ethnicity.

\section{Conclusion}

Severe lymphopenia was associated with reduced overall survival and progression-free survival in Hispanic women undergoing definitive chemoradiation for cervical cancer, but not associated with outcomes in non-Hispanic women.

Keywords: Uterine cervical neoplasms; Chemoradiotherapy; Anemia; Lymphopenia

\section{Introduction}

In the United States, approximately 13,000 women are diagnosed with cervical cancer yearly, over $30 \%$ of whom will perish from their disease [1]. For those diagnosed with locoregionally advanced carcinoma, cisplatin-based chemoradiation therapy is considered the standard of care [2]. Though effective, chemoradiation is frequently complicated by hematologic toxicities that increase the risk of adverse events and limit a patient's ability to complete her treatment regimen [3]. Lymphocytes are particularly susceptible to chemoradiation,
Received: 2021.10.04. Revised: 2022.01.19. Accepted: 2022.02.03. Corresponding author: Michelle S Ludwig, MD, PhD Department of Radiation Oncology, Baylor College of Medicine, 1 Baylor Plaza, Houston, TX 77030, USA E-mail: michelle.ludwig@bcm.edu https://orcid.org/0000-0001-8860-7269

*These authors contributed equally to this work.

Articles published in Obstet Gynecol Sci are open-access, distributed under the terms of the Creative Commons Attribution Non-Commercial License (http://creativecommons. org/licenses/by-nc/3.0/) which permits unrestricted non-commercial use, distribution, and reproduction in any medium, provided the original work is properly cited.

Copyright $\odot 2022$ Korean Society of Obstetrics and Gynecology 


\section{Obstetrics \& Gynecology Science}

Feiya Shi, et al. CRT heme toxicities in cervical cancer

with decreased cell counts seen following radiation treatment with and without chemotherapy [4]. An adverse impact of chemoradiation therapy on erythrocyte production is also well-documented and often results in transfusion-dependent anemia and worsened quality of life $[5,6]$.

As interest grows regarding the body's physiological response to cancer, these non-cancerous cells of the tumor microenvironment have been implicated in the pathogenesis of tumor progression and treatment resistance. Of immune cells involved in the body's antitumor response, CD $8^{+} \mathrm{T}$ lymphocytes are known to play a critical role in tumor destruction via enzymatic cell lysis and cytostatic lymphokine production [7]. In melanoma, colorectal, breast and ovarian cancers, the presence of tumor-infiltrating lymphocytes in tumor stroma has been linked to slower rates of tumor growth and improved outcomes $[8,9]$. Both pre- and post-treatment lymphopenia have been found to correlate with poor survival outcomes in both gynecologic and non-gynecologic malignancies [10-12]. A number of studies have also examined the detrimental effect of intra-treatment lymphopenia, reporting low absolute lymphocyte count (ALC) during chemoradiation therapy to be predictive of progression-free survival and disease-specific survival in various cancers [13-17].

Anemia is common during chemoradiation treatment and may arise for numerous reasons: insufficient red blood cell production secondary to nutritional deficiencies, hemolysis, myelosuppression, etc. [18]. Similar to lymphopenia, the presence of anemia has been associated with poor outcomes in a variety of cancers [19]. In cervical cancer, studies have found low hemoglobin $[\mathrm{Hg}]$ level before and during radiotherapy to be strongly prognostic for local control and survival $[20,21]$. Though the relationship between anemia and poor prognosis is unclear, one explanation is that anemic hypoxia impairs the production of cytotoxic free radicals, limiting the extent that radiation therapy can reduce tumor size [22]. More recently, however, a number of reports have questioned the potential causality of this relationship $[23,24]$.

Chemoradiation-induced hematologic toxicity may contribute to worsened locoregional disease control and poor treatment outcomes for women diagnosed with cervical cancer. Though prior studies have examined the individual impact of lymphopenia and anemia on cervical cancer outcomes, there is limited data regarding the prognostic significance of concomitant hematologic toxicities during chemoradiation therapy. Here, we sought to identify risk factors for the development of hematologic toxicities during cervical cancer treatment and examine the prognostic predictive value of these toxicities alone and in combination on overall survival (OS), progression-free survival (PFS), and local control (LC).

\section{Materials and methods}

After institutional review board approval, demographic data was abstracted from all patients treated at an urban safetynet hospital for invasive cervical carcinoma between January 1, 2012 and December 31, 2015. Patients were considered to be eligible if they had biopsy-proven cervical cancer that was treated with definitive chemoradiation or surgery followed by definitive chemoradiation. Definitive chemoradiation was defined as receiving definitive intent radiation to at least 45 Gy with concurrent cisplatin. All patients were scheduled for brachytherapy, though not all patients completed their treatment course.

Data regarding hematologic variables, disease characteristics, and treatment course were retrospectively abstracted. Patients were staged per International Federation of Gynecology and Obstetrics (FIGO) 2009 guidelines. Any women diagnosed with stage IV disease were excluded from further analysis, as they are generally treated with neoadjuvant chemotherapy prior to radiation at our institution. Clinical baseline was defined as the 4-week window prior to the administration of the first dose of chemo- or pelvic radiotherapy. Hematologic variables $(\mathrm{Hg}$, white blood cell count, platelet count, ALC, and absolute neutrophil count) were collected at clinical baseline, during weeks 1-6 of concurrent chemoradiation, and at first follow-up appointment after completion of all radiation and chemotherapy. The nadir for each hematologic variable during weeks 1-6 of concurrent chemoradiation therapy was used to define the grade of toxicity experienced for each cell line. Toxicities were defined as per the National Cancer Institute's Common Terminology Criteria for Adverse Events (CTCAE) version 4.03. Followup was conducted every 3 months with physical and vaginal speculum exam, imaging, and biopsy if clinically indicated.

Associations between hematologic variables and clinical outcomes were evaluated using Kaplan-Meier and Cox regression analyses. Hematologic toxicities including anemia, leukopenia, thrombocytopenia, neutropenia, and lymphopenia were analyzed. For initial univariate Kaplan-Meier and 


\section{Obstetrics \& Gynecology Science}

Vol. 65 , No. 2, 2022

Cox analyses, patients were partitioned based on highest toxicity grade at nadir during treatment, and groups were compared sequentially based on grade using a reverse partition analysis to find the most clinically relevant toxicities. OS, LC, and PFS were analyzed. Length of follow-up was defined from date of initial biopsy positive for cervical cancer to date of last known contact or date of death. Progression date was defined as date of positive biopsy, if available. If unavailable, then date of imaging showing progression was used. LC was defined as any recurrence in the pelvis, while PFS included local or distant recurrence.

Univariate Cox regression analysis was used to evaluate other patient and disease characteristics that could be associated with outcomes, including chemotherapy dose (both in number of doses as well as overall dose received), length of radiation (in days), the use of extended fields, age, FIGO stage, histology, and presence of comorbidities. Multivariable Cox regression analysis was performed using all variables known to be prognostic factors in the literature, and for those in our dataset with $P<0.25$ in univariate analyses. All variables were included in the same multivariable model, including the hematologic variables found to be significantly associated with outcomes. Variables were excluded if the model did not converge with their inclusion.

Logistic regression was then performed to look at variables associated with the hematologic toxicities that had been found to be associated with survival and recurrence outcomes. All analyses were done using Statistical Package for the Social Sciences (SPSS) version 24.0 (IBM Corp, Armonk, NY, USA). In accordance with the journal's guidelines, we will provide our data for the reproducibility of this study in other centers if such is requested.

\section{Results}

After querying the institutional database for women diagnosed with cervical cancer treated between 2012 and 2015, and removing patients with stage IV disease, we identified a total of 121 subjects suitable for inclusion. Median followup for these women was 34.0 months (95\% confidence interval, 30.8-37.2). Median OS time was not reached, with 17 patients passing away during follow-up. The majority of subjects were Hispanic ( $n=83,68.6 \%)$. Median age at diagnosis was 45 years old (interquartile range, 40-52) (Table 1).
Most were diagnosed with squamous cell carcinoma ( $n=101$, 83.5\%), and clinical FIGO 2009 stage was well distributed across stage I $(n=46,38.0 \%)$, stage II $(n=47,38.8 \%)$, and stage III $(n=28,23.1 \%)$ disease. The majority of patients were treated with definitive chemoradiation, though 18 $(14.9 \%)$ were treated post-operatively. Seven patients (5.8\%) had been previously diagnosed with human immunodefi-

Table 1. Patient demographics and disease characteristics

\begin{tabular}{|c|c|}
\hline & Value $(n=121)$ \\
\hline Age at diagnosis & $45(40-52)$ \\
\hline \multicolumn{2}{|l|}{ Race/ethnicity } \\
\hline Non-hispanic & $38(31.4)$ \\
\hline Hispanic & $83(68.6)$ \\
\hline BMl & $29.59 \pm 6.85$ \\
\hline \multicolumn{2}{|l|}{ Histology } \\
\hline SCC & $101(83.5)$ \\
\hline Adenosquamous & $5(4.1)$ \\
\hline Adenocarcinoma & $14(11.6)$ \\
\hline Serous adenocarcinoma & $1(0.8)$ \\
\hline \multicolumn{2}{|l|}{ Stage } \\
\hline IB & $46(38.0)$ \\
\hline$\| A$ & $11(9.1)$ \\
\hline$\| B$ & $36(29.8)$ \\
\hline$\| \mathrm{A}$ & $2(1.7)$ \\
\hline$\| I I B$ & $26(21.5)$ \\
\hline Diabetes & $13(10.7)$ \\
\hline HIV & $7(5.8)$ \\
\hline Post-operative & $18(14.9)$ \\
\hline Length of XRT (days) & $59.8 \pm 20.3 ; 57(49-65)$ \\
\hline Radiation $>56$ days & $64(52.90)$ \\
\hline External beam dose (Gy) & $5,588 \pm 1,236 ; 5,356(4,700-6,175)$ \\
\hline Brachytherapy dose (Gy) & $3,232 \pm 1,430 ; 3,943(2,040-4,190)$ \\
\hline \multicolumn{2}{|l|}{ Radiation Modality } \\
\hline $3 D$ & $56(46.3)$ \\
\hline IMRT & $65(53.7)$ \\
\hline Use of extended fields & $48(39.7)$ \\
\hline At least six cisplatin cycles & $50(41.3)$ \\
\hline Total cisplatin dose (mg) & $340(78.4)$ \\
\hline
\end{tabular}

Values are presented as number (\%), median (interquartile ranges), or mean \pm standard deviation.

BMI, body mass index; SCC, squamous cell carcinoma; XRT, radiotherapy; IQR, interquartile range; 3D, three-dimensional; IMRT, intensity-modulated radiation therapy. 


\section{Obstetrics \& Gynecology Science}

Feiya Shi, et al. CRT heme toxicities in cervical cancer

ciency virus (HIV) infection at time of cancer diagnosis. There was an equal distribution of patients who received 3D-conformal radiation or intensity modulated radiation therapy to their primary disease site $(n=56,46.3 \%$ and $n=65,53.7 \%$, respectively). There were 48 patients (39.75\%) who had extended field radiation that included the para-aortic nodes. Only $41.3 \%(n=50)$ of patients received six or more cycles of cisplatin (nine patients received more than six cycles due to a protracted radiation treatment course), with mean cumulative cisplatin dose given over the course of treatment of $340.8 \pm 78.4 \mathrm{mg}$.

All patients experienced hematologic toxicities during chemoradiation (Fig. 1). Every patient had a minimum of grade 2 lymphopenia, with the majority experiencing grade $3(n=70,58.7 \%)$ or grade $4(n=44,36.4 \%)$ lymphocyte toxicity. The rarest toxicity was neutropenia, which was experienced by only $24 \%$ of patients $(n=29)$. Most subjects had some degree of anemia $(n=111,91.7 \%)$ and leukopenia $(n=103,85.1 \%)$, while thrombocytopenia occurred less frequently $(n=41,33.9 \%)$. With recursive partition analysis, we found that grade 4 lymphopenia and grade 3-4 anemia were the hematologic toxicities most associated with outcomes (Fig. 2). On univariate cox regression, grade 4 lymphopenia was significantly associated with OS (hazard ratio [HR], 4.455; $P=0.005)$, PFS (HR, 3.422; $P=0.001)$, and $L C(H R$, 4.077; $P=0.047$ ) (Table 2). Grade 3, 4 anemia was associated with OS (HR,4.086; $P=0.014)$, though not PFS (HR, 1.795; $P=0.275)$. There were too few sentinel events to analyze the association between grade 3, 4 anemia and LC.

On univariate Cox regression, age was associated with OS, though not PFS or LC (HR, 2.065; $P=0.008$; HR, 1.03; $P=0.097$; and $H R=0.995 ; P=0.881$, respectively). Prior diagnosis of HIV infection approached significance for LC $(H R, 4.648 ; P=0.056)$. Stage III disease was associated with worse OS, PFS, and LC (HR, 10.323; $P=0.003 ; H R, 7.029$; $P<0.001$; and HR, 5.499; $P=0.05$, respectively). Patients requiring extended field radiation had worse PFS (HR, 3.709; $P<0.001)$, though neither OS nor $L C$ reached statistical significance (HR, 2.45; $P=0.07 ; H R, 3.793 ; P=0.06$, respectively). Increased duration of treatment (due to missed radiation

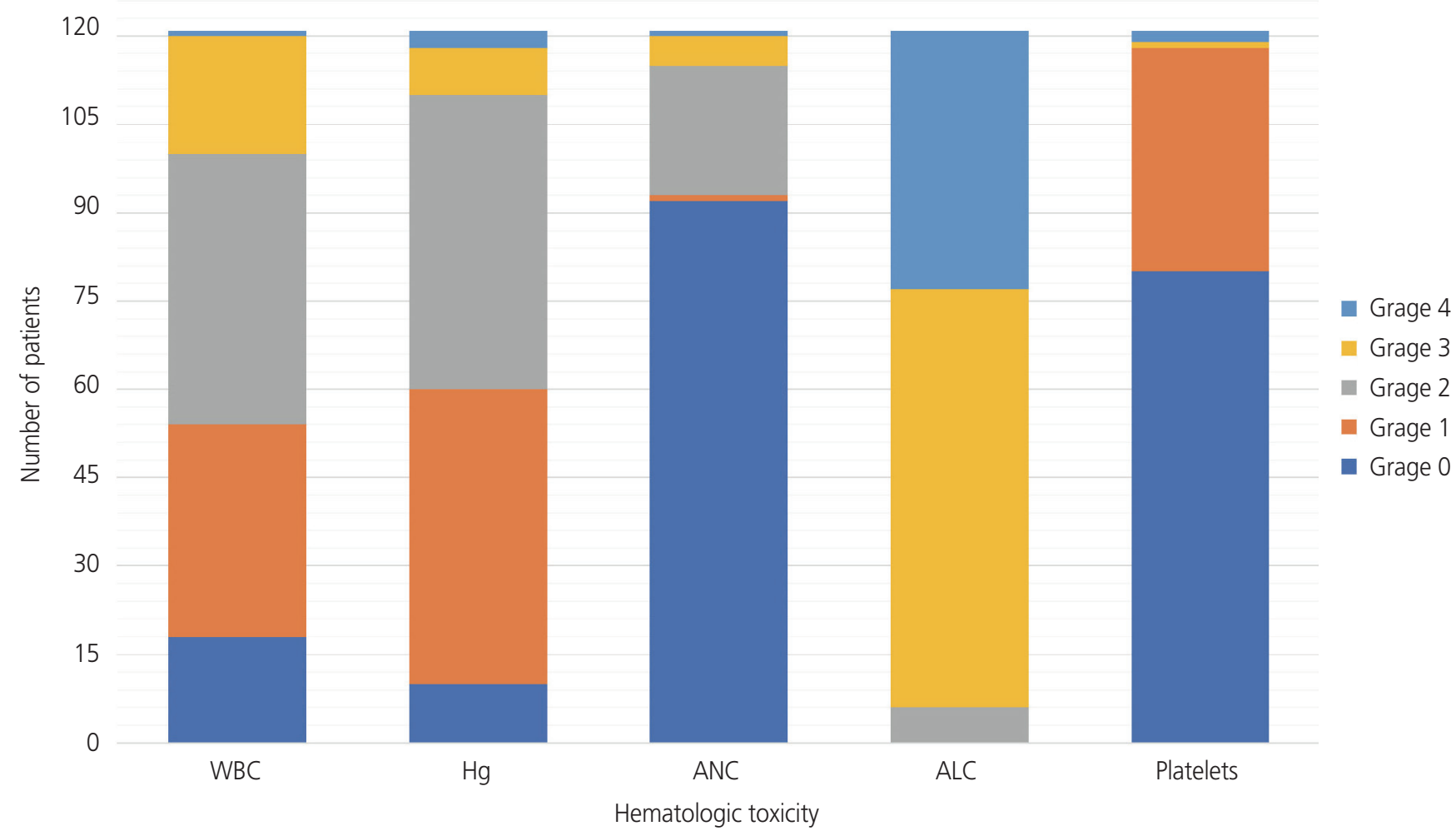

Fig. 1. Hematologic toxicity at nadir during concurrent chemoradiation. WBC, white blood cell; Hg, hemoglobin; ANC, absolute neutrophil count; ALC, absolute lymphocyte count. 


\section{Obstetrics \& Gynecology Science}

Vol. 65, No. 2, 2022

doses) was associated with reduced OS and PFS, though not LC (HR, 1.018; $P=0.003 ; H R, 1.019 ; P=0.011$; and HR, 1.007; $P=0.753)$. Patients treated post-operatively did not have different outcomes than those treated definitively (all $P>0.05$ ). Increased $\mathrm{Hg}$ and $\mathrm{ALC}$ at baseline trended towards improved outcomes. Increased $\mathrm{Hg}$ was significantly associated with improved PFS and LC (HR, 0.718; $P<0.001 ; H R, 0.700$;
$P=0.036)$, and increased ALC was significantly associated with improved LC (HR, 0.271; $P=0.044)$.

Multivariable cox regression analysis was done analyzing both grade 4 lymphopenia and grade 3, 4 anemia while controlling for other confounders (Table 3). Stage, total cisplatin dose, age, the presence of HIV, the use of extended fields, increased length of radiation treatment, and baseline ALC and
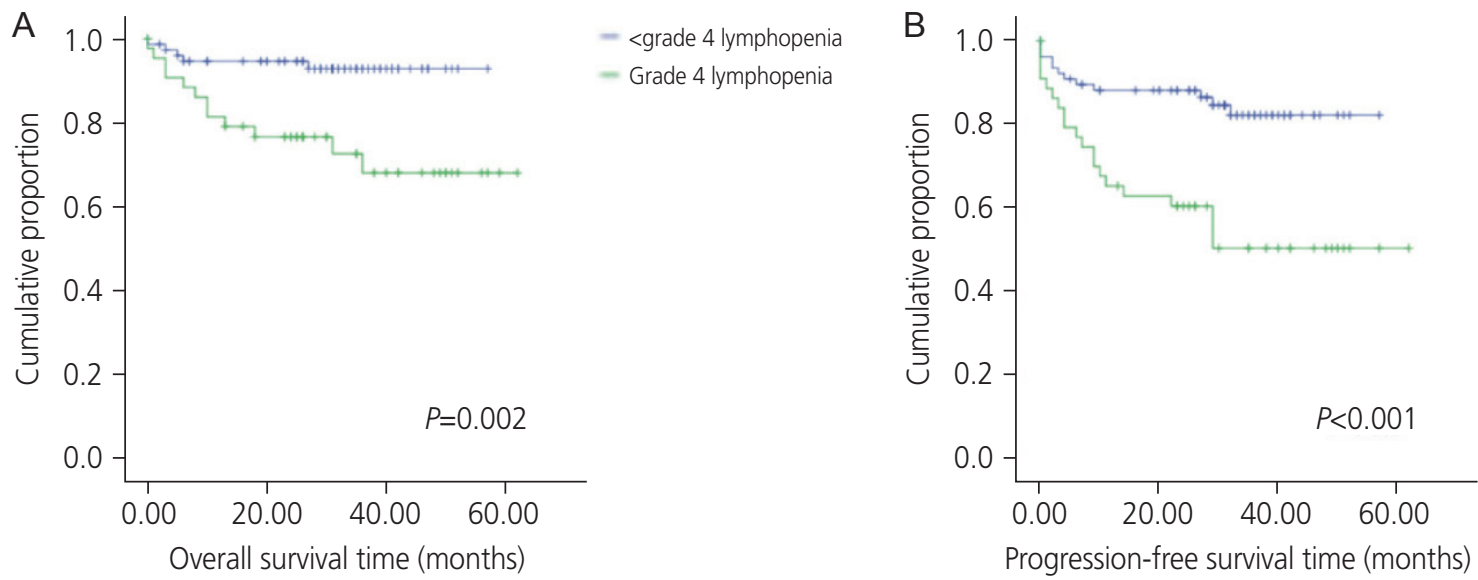

- <grade 4 lymphopenia

Progression-free survival time (months)
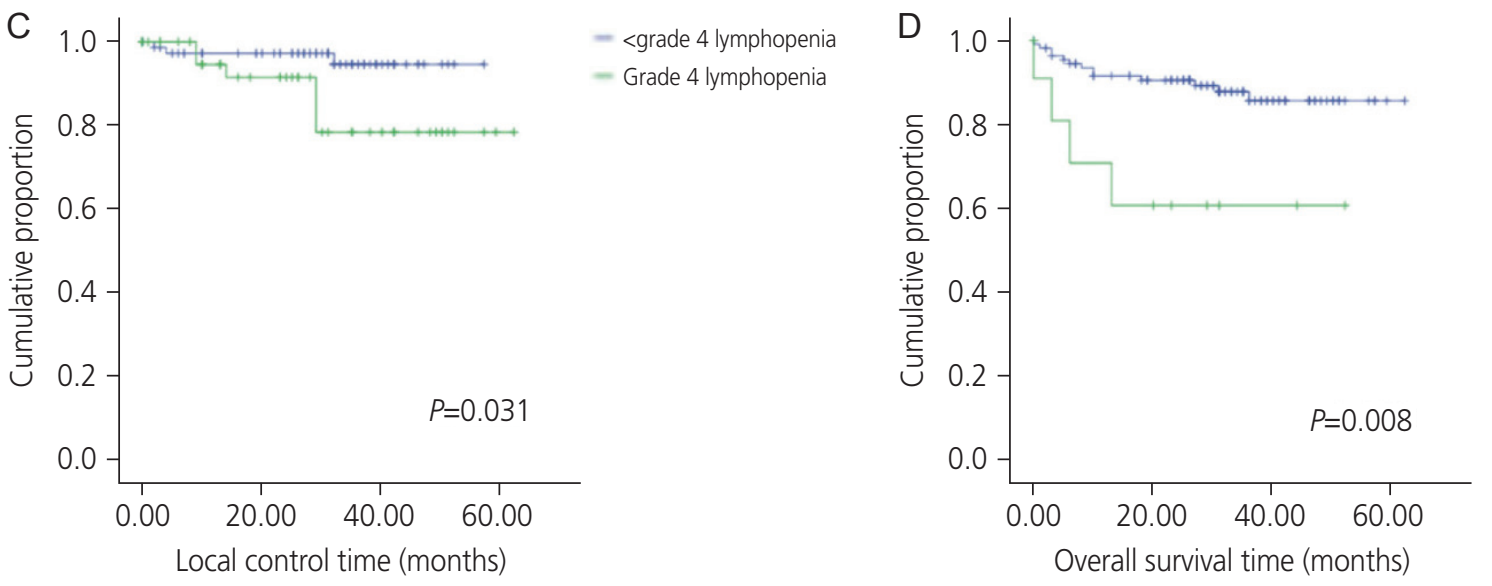

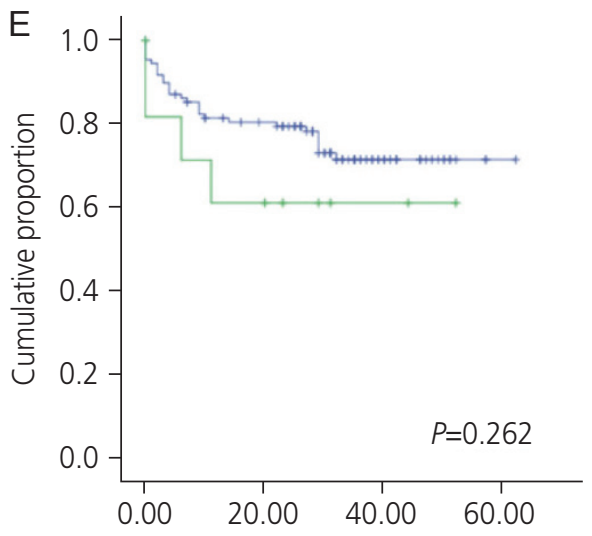

Progression-free survival time (months)
- <grade 3 anemia

- Grade 3 to 4 anemia
- <grade 3 anemia

- Grade 3 to 4 anemia

Fig. 2. Kaplan-Meier curve showing OS, PFS, and LC for patients with grade 4 lymphopenia compared to all other patients (A-C), and OS and PFS for patients with grades 3 to 4 anemia compared to those with grades 0 to 2 anemia $(D, E)$. OS, overall survival; PFS, progression-free survival; LC, local control. 


\section{Obstetrics \& Gynecology Science}

Feiya Shi, et al. CRT heme toxicities in cervical cancer

Table 2. Univariate cox regression analysis comparing OS, PFS, and LC for patients with hematologic toxicities

\begin{tabular}{|c|c|c|c|c|c|c|}
\hline & \multicolumn{2}{|c|}{ Overall survival } & \multicolumn{2}{|c|}{ Progression-free survival } & \multicolumn{2}{|c|}{ Local control } \\
\hline & HR & $P$-value & HR & $P$-value & HR & $P$-value \\
\hline Age & 1.065 & 0.008 & 1.03 & 0.097 & 0.995 & 0.881 \\
\hline \multicolumn{7}{|l|}{ Ethnicity } \\
\hline Non-hispanic & 1 & Ref & 1 & Ref & 1 & Ref \\
\hline Hispanic & 1.041 & 0.939 & 0.940 & 0.871 & 0.796 & 0.748 \\
\hline BMI & 0.966 & 0.388 & 0.956 & 0.129 & 0.89 & 0.078 \\
\hline HIV & 1.08 & 0.940 & 1.578 & 0.453 & 4.648 & 0.056 \\
\hline \multicolumn{7}{|l|}{ Stage } \\
\hline । & 1 & Ref & 1 & Ref & 1 & Ref \\
\hline$\|$ & 3.138 & 0.161 & 1.849 & 0.234 & 1.701 & 0.561 \\
\hline III & 10.323 & 0.003 & 7.029 & $<0.001$ & 5.499 & 0.05 \\
\hline Post-operative & 0.038 & 0.260 & 0.038 & 0.116 & 0.038 & 0.404 \\
\hline At least six cycles of cisplatin & 0.416 & 0.125 & 0.512 & 0.089 & 0.394 & 0.246 \\
\hline Cisplatin dose (mg) & 0.994 & 0.039 & 0.995 & 0.011 & 0.995 & 0.188 \\
\hline Extended fields & 2.45 & 0.07 & 3.709 & $<0.001$ & 3.793 & 0.06 \\
\hline Increased length of XRT & 1.018 & 0.003 & 1.019 & 0.001 & 1.007 & 0.753 \\
\hline Baseline ALC & 0.688 & 0.321 & 0.637 & 0.104 & 0.271 & 0.044 \\
\hline Baseline $\mathrm{Hg}$ & 0.808 & 0.092 & 0.718 & $<0.001$ & 0.700 & 0.036 \\
\hline Grade 4 lymphopenia & 4.455 & 0.005 & 3.422 & 0.001 & 4.007 & 0.047 \\
\hline Grade 3 to 4 anemia & 4.086 & 0.014 & 1.795 & 0.275 & - & - \\
\hline
\end{tabular}

OS, overall survival; PFS, progression-free survival; LC, local control; HR, hazard ratio; Ref, reference; BMI, body mass index; HIV, human immunodeficiency virus; XRT, radiotherapy; ALC, absolute lymphocyte count; Hg, hemoglobin.

Table 3. Multivariable cox regression analysis comparing OS, PFS, and ALC for patients with hematologic toxicities

\begin{tabular}{|c|c|c|c|c|c|c|}
\hline & \multicolumn{2}{|c|}{ Overall survival } & \multicolumn{2}{|c|}{ Progression-free survival } & \multicolumn{2}{|c|}{ Local control } \\
\hline & HR & $P$-value & HR & $P$-value & HR & $P$-value \\
\hline Age & 1.052 & 0.081 & 1.041 & 0.050 & 0.986 & 0.717 \\
\hline HIV & 0.957 & 0.969 & 1.684 & 0.416 & 4.945 & 0.086 \\
\hline \multicolumn{7}{|l|}{ Stage } \\
\hline । & 1 & Ref & 1 & Ref & 1 & Ref \\
\hline$\|$ & 1.598 & 0.591 & 1.630 & 0.366 & 2.098 & 0.476 \\
\hline III & 1.495 & 0.659 & 2.326 & 0.144 & 3.194 & 0.268 \\
\hline Total cisplatin dose (mg) & 0.990 & 0.006 & 0.995 & 0.020 & 0.998 & 0.596 \\
\hline Extended fields & 0.750 & 0.797 & 1.817 & 0.173 & 2.817 & 0.204 \\
\hline Increased length of XRT & 1.036 & $<0.001$ & 1.021 & $<0.001$ & 0.995 & 0.918 \\
\hline Baseline ALC & 0.799 & 0.580 & 0.835 & 0.557 & 0.324 & 0.146 \\
\hline Baseline $\mathrm{Hg}$ & 0.973 & 0.898 & 0.651 & 0.006 & 0.730 & 0.169 \\
\hline Grade 4 lymphopenia & 9.850 & 0.007 & 2.082 & 0.105 & 2.862 & 0.210 \\
\hline Grade 3 to 4 anemia & 11.495 & 0.023 & 0.471 & 0.309 & - & - \\
\hline
\end{tabular}

OS, overall survival; PFS, progression-free survival; ALC, absolute lymphocyte count; HR, hazard ratio; HIV, human immunodeficiency virus; Ref, reference; XRT, radiotherapy; ALC, absolute lymphocyte count; Hg, hemoglobin. 


\section{Obstetrics \& Gynecology Science}

Vol. 65, No. 2, 2022

$\mathrm{Hg}$ were included as co-variables. Post-operative versus definitive treatment was originally included in the model, however, likely due to low $n$, did not converge for any outcome so was removed. In multivariable analysis, both lymphopenia and anemia during treatment remain independently associated with OS after adjusting for confounding variables and the presence of the other $(H R, 9.850 ; P=0.007 ; H R, 11.495$; $P=0.023$, respectively). Neither were associated with PFS or LC. Baseline ALC was not associated with any outcome on multivariable analysis, though baseline $\mathrm{Hg}$ remained significantly associated with PFS (HR, 0.651; $P=0.006)$. Clinical stage, patient age, and prior diagnosis with HIV infection were not significant for any outcome. Increased length of radiation was significantly associated with reduced $O S$ and PFS (HR, 1.035; $P<0.001 ; H R, 1.022 ; P<0.001$, respectively), though not LC (HR, 2.991; $P=0.166)$.

We then examined the effect of ethnicity and found that the effect of grade 4 lymphopenia on survival outcomes

Table 4. Effect modification of ethnicity on the effect of hematologic toxicities

\begin{tabular}{|c|c|c|c|c|c|c|}
\hline & \multicolumn{2}{|c|}{ Overall survival } & \multicolumn{2}{|c|}{ Progression-free survival } & \multicolumn{2}{|c|}{ Local control } \\
\hline & HR & $P$-value & HR & $P$-value & HR & $P$-value \\
\hline Hispanic grade 4 lymphopenia & 5.514 & 0.010 & 4.288 & 0.094 & 4.640 & 0.001 \\
\hline Non-hispanic grade 4 lymphopenia & 2.701 & 0.277 & 1.765 & 0.369 & 3.479 & 0.309 \\
\hline Hispanic grade $>3$ anemia & 7.999 & 0.001 & 3.351 & 0.031 & 0.046 & 0.718 \\
\hline Non-hispanic grade $>3$ anemia & 0.041 & 0.614 & 0.040 & 0.461 & 0.039 & 0.652 \\
\hline
\end{tabular}

HR, hazard ratio.

Table 5. Risk factors associated with hematologic toxicities

\begin{tabular}{|c|c|c|c|c|}
\hline & \multicolumn{2}{|c|}{ Grade 4 ALC } & \multicolumn{2}{|c|}{ Grade 3 to $4 \mathrm{Hg}$} \\
\hline & OR & $P$-value & OR & $P$-value \\
\hline Age & 1.018 & 0.318 & 1.019 & 0.533 \\
\hline \multicolumn{5}{|l|}{ Ethnicity } \\
\hline Non-hispanic & 1 & Ref & - & - \\
\hline Hispanic & 1.031 & 0.941 & - & - \\
\hline BMI & 0.928 & 0.025 & 0.922 & 0.168 \\
\hline HIV diagnosis & 2.3 & 0.291 & 1.65 & 0.658 \\
\hline Diabetes diagnosis & 0.49 & 0.3 & 0.49 & 0.999 \\
\hline \multicolumn{5}{|l|}{ Histology } \\
\hline Other & 1 & Ref & 1 & Ref \\
\hline SCC & 3.872 & 0.04 & 2.088 & 0.495 \\
\hline \multicolumn{5}{|l|}{ Stage } \\
\hline I & 1 & Ref & - & - \\
\hline$\|$ & 2.04 & 0.128 & - & - \\
\hline III & 5.564 & 0.001 & - & - \\
\hline Post-operative & 0.855 & 0.772 & - & - \\
\hline Length of XRT (days) & 1.004 & 0.663 & 1.005 & 0.685 \\
\hline Use of Extended fields & 2.055 & 0.064 & 1.279 & 0.699 \\
\hline Baseline ALC & 0.338 & 0.001 & - & - \\
\hline Baseline $\mathrm{Hg}$ & - & - & 0.286 & $<0.001$ \\
\hline
\end{tabular}

ALC, absolute lymphocyte count; Hg, hemoglobin; OR, overall risk; BMI, body mass index; HIV, human immunodeficiency virus; Ref, reference; $X R T$, radiotherapy. 


\section{Obstetrics \& Gynecology Science}

Feiya Shi, et al. CRT heme toxicities in cervical cancer

was only seen with Hispanic patients. OS and LC were significantly associated with grade 4 lymphopenia (HR, 5.514; $P=0.010 ; H R, 4.640 ; P=0.001)$, while PFS approached significance (HR, 4.288; $P=0.094)$. Grade 3,4 anemia was also associated with OS in Hispanic patients (HR, 7.999; $P=0.001$ ) and PFS (HR, 3.351; $P=0.031)$. However, this was not true for non-Hispanic patients, for whom neither grade 4 lymphopenia nor grade 3, 4 anemia was associated with any outcome (all $P>0.05$ ) (Table 4). Patients of Hispanic ethnicity were not more likely to have grade 4 lymphopenia during treatment than non-Hispanic patients ( $36.1 \%$ vs. $36.8 \%, P>0.99$ ), nor more likely to have grade 3, 4 anemia (9.6\% vs. $7.9 \%$, $P>0.99)$ (data not shown). They were also not more likely to be diabetic (10.8\% vs. $10.5 \%, P>0.99)$. However, they were less likely to have HIV than non-Hispanic patients (1.2\% vs. $15.8 \%, P=0.006)$.

The only variable that was significantly associated with the presence of grades 3, 4 anemia on logistic regression was baseline Hf (overall risk [OR], 0.286; $P<0.001$ ) (Table 5). The same relationship remained with lymphopenia, in that baseline ALC was significantly associated with grade 4 lymphopenia ( $O R, 0.338 ; P=0.001)$. In addition, patients with higher body mass index (BMI) were less likely to have grade 4 lymphopenia during treatment $(O R, 0.928 ; P=0.025)$. Analysis also found that those with stage III disease were more likely to have grade 4 lymphopenia (OR, 5.564; $P=0.001)$, and that those with squamous cell histology were more likely to see this toxicity (OR, 3.782; $P=0.04)$. HIV was not associated with either grade 4 lymphopenia nor grade 3, 4 anemia (OR, 2.3; $P=0.291 ; O R, 1.65 ; P=0.658$, respectively).

Patients with grade 4 lymphopenia had a reduced cisplatin dose (mean dose, $320.2 \mathrm{mg}$; standard deviation [SD], 73.2) compared to those who did not experience grade 4 lymphopenia (mean dose, 351.4 mg; SD, 79.5; $P=0.035$ ). They were not more likely, however, to receive fewer total cisplatin cycles ( $P=0.10$, data not shown). While total dose of cisplatin was associated with statistically decreased OS (HR, 0.99; $P=0.006)$, and decreased PFS (HR, 0.995; $P=0.020)$, clinically these differences are negligible.

\section{Discussion}

Our study examined various hematologic toxicities during chemoradiation therapy for cervical cancer by quantifying cell count nadir throughout treatment. Hematologic toxicities varied in distribution of prevalence and severity. Of the toxicities, lymphopenia and anemia were both common and significantly associated with worse outcomes, whereas neutropenia and thrombocytopenia were neither common nor prognostic. Altogether, these results highlight the importance of lymphocyte and erythrocyte function in mounting response to antitumor therapy and deemphasize the significance of other concomitant toxicities.

Low lymphocyte count during treatment has been previously established as a poor prognostic factor in several cancers, including cervical cancer $[10,25,26]$. Bogani et al. [26] found that grade 2+ leukopenia during chemoradiation for cervical cancer was associated with reduced OS, while a study by Wu et al. [10] found that both pre- and posttreatment leukopenia was associated with decreased survival, though statistical significance was not reached. Kitayama et al. [13] found that circulating lymphocyte number during chemoradiation therapy was positively associated with tumor response in rectal cancer. Treatment-related lymphopenia has been shown to be predictive for survival outcomes in head and neck, pancreatic and lung cancers $[14,16,17]$. Davuluri et al. [15] further studied this phenomenon in esophageal, finding grade 4 lymphopenia during chemoradiation therapy to be particularly associated with poor outcomes. Taking a similar standardized approach, we found grade 4 lymphopenia to be strongly associated with survival in cervical cancer, even after controlling for patient characteristics, treatment factors, including total cisplatin dose, and baseline ALC. With growing evidence for the link between severe treatmentrelated lymphopenia and poor prognosis, our results support the idea that optimal treatment outcomes in cervical cancer are at least partially dependent on adequate host immune response involving lymphocytes.

The exact mechanisms by which lymphopenia contributes to worsened cancer survival outcomes remains unclear. It may be that individuals with poor overall health status are more prone to developing lymphopenia, though several studies have shown that lymphopenia independently predicts for decreased cancer survival even after considering functional status or infection-related mortality [27]. Since the presence of tumor-infiltrating lymphocytes has been linked to cancer survival $[8,9]$, it is possible that such lymphocytes mediate initial recognition of tumor-associated antigens, inhibition of tumor migration, and promotion of antitumor apoptosis 


\section{Obstetrics \& Gynecology Science}

Vol. 65 , No. 2, 2022

and cytotoxicity, all vital to the body's intrinsic anticancer response. Based on our study of lymphopenia and cervical cancer outcomes, we hypothesize that women who better retain lymphocyte numbers throughout treatment are able to mount a more robust immune response against carcinogenesis, thereby prolonging survival.

Interestingly, the odds of developing grade 4 lymphocyte toxicity were correlated with patient and disease characteristics, namely baseline ALC, BMI, stage III disease, and squamous cell histology, though not treatment variables such as length of radiation or use of extended fields. However, length of radiation and use of extended fields were each independently associated with worse survival outcomes on univariate analysis, indicating of course that treatmentassociated morbidity and mortality cannot be fully explained by lymphocyte loss during chemoradiation therapy. Paraaortic lymph node involvement requiring the use of extended field radiation, as well as increase in treatment duration, are known to be associated with poor outcomes [28-30]. Furthermore, these results suggest that multiple factors outside of the standard chemoradiation protocols for cervical cancer predispose patients to severe lymphopenia in the first place, including but not limited to baseline ALC.

Overall cisplatin dose was lower in those with grade 4 lymphopenia, indicating dose reductions; however, they were not more likely to miss chemotherapy cycles than those without grade 4 lymphopenia. This is multifactorial and can be due to the presence of other hematologic toxicities requiring a chemotherapy pause, such as neutropenia, or other toxicities in general, including ototoxicity. While total cisplatin dose was statistically associated with survival (HR, 0.990; $P=0.006)$, this number is not clinically significant. This lends to the conclusion that lymphopenia itself contributes to survival independent of chemotherapy, which correlates with findings from other institutions $[25,31,32]$.

Previous studies have shown associations between race and hematologic toxicities during treatment, which have affected survival outcomes [33]. On our analysis, we found similar results in that grade 4 lymphopenia was associated with poor outcomes in Hispanic patients but this effect was not seen in non-Hispanic patients. The only differing patient characteristic we found was that Hispanic patients were less likely to be HIV positive than non-Hispanic patients in our cohort. While HIV positivity itself was not associated with grade 4 lymphopenia nor OS, there is perhaps an interaction between the presence of HIV and the effect of grade 4 lymphopenia in outcomes. Alternatively, the definitions for hematologic toxicities may need to be adjusted depending on ethnicity. In a study done by Azab et al. [33], they found that Hispanic and non-Hispanic black patients had lower neutrophil to lymphocyte ratios at baseline than non-Hispanic white patients, indicating that perhaps different cut-off points are needed when examining the effect of hematologic variables on various disease outcomes.

High BMI was associated was decreased likelihood of severe lymphopenia. This association has been reported in the use of systemic treatment for multiple sclerosis [34], though to our knowledge has not yet been reported in patients undergoing radiation treatment. The reason for this association is unclear. It may be due to baseline characteristics, and that patients with higher BMI are less likely to become sarcopenic, which has been shown to be associated with high neutrophil to lymphocyte ratio [35]. Another possible explanation is that higher BMI indicates larger bone marrow volume, which has been shown to be protective against hematologic toxicities during pelvic radiation [36].

In cervical cancer patients, the strong correlation between anemia and tumor recurrence has been well-documented, hypoxia-induced radiation resistance being the most commonly cited mechanism [24]. A number of studies have suggested that the presence of anemia before and/or during treatment portends poor prognosis. Though previous reports also measured $\mathrm{Hg}$ nadir during treatment, few studies have defined a standardized $\mathrm{Hg}$ level that was prognostic for poor outcomes. Using CTCAE criteria, we found grade 3, 4 anemia during chemoradiation therapy to be significantly associated with OS. None of the prognostic or treatment factors we analyzed were found to be associated with the presence of grade 3, 4 anemia on logistic regression other than baseline $\mathrm{Hg}$.

Our study was not without limitations. As our study design was retrospective, we were unable to account for all factors that could influence cell count during treatment. While some reports have found bone marrow dose to correlate with incidence and severity of hematologic toxicities $[15,37,38]$, we did not include bone marrow data as a treatment variable in our analysis. Not much variability is seen in our bone marrow dose between patients, as the institutional practice is to limit mean bone marrow dose to $35 \mathrm{~Gy}$, and the dose rarely approaches this value. Bone marrow dose was not available for 


\section{Obstetrics \& Gynecology Science}

Feiya Shi, et al. CRT heme toxicities in cervical cancer

all patients, though was included as a test variable for the patients in which it was available, and it was not associated with clinical outcomes or hematologic toxicities. We also included both patients who were treated with post-operative as well as definitive chemoradiation for their cervical cancer. While these populations can vary in their baseline risk for local recurrence and OS, we did not find a significant difference between the two in this cohort, likely due to the small number of patients in the post-operative group. In addition, this variable was not associated with either grade 4 lymphopenia or grade 3, 4 anemia. As such, we elected to include both in this analysis.

Despite these limitations, our findings on the prognostic importance of severe lymphopenia and anemia during chemoradiation therapy may help to guide clinical thought processes and investigation regarding treatment optimization in cervical cancer. Recently, immuno-oncology has emerged as a potential novel approach for women with node-positive locally advanced and metastatic cervical cancer disease who respond poorly to chemoradiation therapy [39]. Given that most cervical cancers are caused by high-risk human papillomavirus strains [40] encoding tumor-specific antigens, targeted immunotherapy may be able to alter the immune microenvironment and bolster host antitumor mechanisms.

In this cohort of women with cervical cancer, the development of grade 4 lymphopenia and grade 3, 4 anemia during chemoradiation therapy was significantly prognostic for poor survival and disease outcomes. As exciting developments such as immune checkpoint inhibition and novel T-cell therapy arise [39], further information about hematologic toxicities in chemoradiation therapy will help to identify patients at risk for standard treatment failure who may be candidates for alternative treatment escalation, including immunotherapeutic options.

\section{Conflict of interest}

No potential conflict of interest relevant to this article was reported.

\section{Ethical approval}

This study does not require approval of the Institutional
Review Board because no patient data is contained in this article. The study was performed in accordance with the principles of the Declaration of Helsinki.

\section{Patient consent}

Written informed consent and the use of images from patients are not required for the publication.

\section{Funding information}

None.

\section{References}

1. Siegel RL, Miller KD, Jemal A. Cancer statistics, 2019. CA Cancer J Clin 2019;69:7-34.

2. Marth C, Landoni F, Mahner S, McCormack M, Gonzalez-Martin A, Colombo N, et al. Cervical cancer: ESMO clinical practice guidelines for diagnosis, treatment and follow-up. Ann Oncol 2017;28(suppl_4):iv72-83.

3. Yang T, Oh J, Apte A, Son CH, Deasy JO, Goodman KA. Predictors of hematologic toxicity during pelvic radiation therapy. Int J Radiat Oncol Biol Phys. 2012;84(3):S346.

4. Abu-Rustum NR, Lee S, Correa A, Massad LS. Compliance with and acute hematologic toxic effects of chemoradiation in indigent women with cervical cancer. Gynecol Oncol 2001;81:88-91.

5. van Meir H, Nout RA, Welters MJ, Loof NM, de Kam ML, van Ham JJ, et al. Impact of (chemo)radiotherapy on immune cell composition and function in cervical cancer patients. Oncoimmunology 2016;6:e1267095.

6. Schrijvers D. Management of anemia in cancer patients: transfusions. Oncologist 2011;16 Suppl 3:12-8.

7. Berke G. The binding and lysis of target cells by cytotoxic lymphocytes: molecular and cellular aspects. Annu Rev Immunol 1994;12:735-73.

8. Clemente CG, Mihm MC Jr, Bufalino R, Zurrida S, Collini $P$, Cascinelli N. Prognostic value of tumor infiltrating lymphocytes in the vertical growth phase of primary cutaneous melanoma. Cancer 1996;77:1303-10.

9. Sato E, Olson SH, Ahn J, Bundy B, Nishikawa H, Qian F, 


\section{Obstetrics \& Gynecology Science}

Vol. 65, No. 2, 2022

et al. Intraepithelial CD8+ tumor-infiltrating lymphocytes and a high CD8+/regulatory $T$ cell ratio are associated with favorable prognosis in ovarian cancer. Proc Natl Acad Sci U S A 2005;102:18538-43.

10. Wu ES, Oduyebo T, Cobb LP, Cholakian D, Kong X, Fader AN, et al. Lymphopenia and its association with survival in patients with locally advanced cervical cancer. Gynecol Oncol 2016;140:76-82.

11. Wild AT, Herman JM, Dholakia AS, Moningi S, Lu Y, Rosati LM, et al. lymphocyte-sparing effect of stereotactic body radiation therapy in patients with unresectable pancreatic cancer. Int J Radiat Oncol Biol Phys 2016;94:571-9.

12. Cézé N, Thibault G, Goujon G, Viguier J, Watier H, Dorval $E$, et al. Pre-treatment lymphopenia as a prognostic biomarker in colorectal cancer patients receiving chemotherapy. Cancer Chemother Pharmacol 2011;68:1305-13.

13. Kitayama J, Yasuda K, Kawai K, Sunami E, Nagawa $\mathrm{H}$. Circulating lymphocyte number has a positive association with tumor response in neoadjuvant chemoradiotherapy for advanced rectal cancer. Radiat Oncol 2010;5:47.

14. Schueneman AJ, Sugar EA, Uram J, Bigelow E, Herman JM, Edil BH, et al. Low total lymphocyte count is associated with poor survival in patients with resected pancreatic adenocarcinoma receiving a GM-CSF secreting pancreatic tumor vaccine. Ann Surg Oncol 2013;20 Suppl 3:S725-30.

15. Davuluri R, Jiang W, Fang P, Xu C, Komaki R, Gomez DR, et al. lymphocyte nadir and esophageal cancer survival outcomes after chemoradiation therapy. Int I Radiat Oncol Biol Phys 2017;99:128-35.

16. Cho O, Oh YT, Chun M, Noh OK, Hoe JS, Kim H. Minimum absolute lymphocyte count during radiotherapy as a new prognostic factor for nasopharyngeal cancer. Head Neck 2016;38 Suppl 1:E1061-7.

17. Campian JL, Ye X, Brock M, Grossman SA. Treatmentrelated lymphopenia in patients with stage III non-smallcell lung cancer. Cancer Invest 2013;31:183-8.

18. Groopman JE, Itri LM. Chemotherapy-induced anemia in adults: incidence and treatment. J Natl Cancer Inst 1999;91:1616-34.

19. Knight K, Wade S, Balducci L. Prevalence and outcomes of anemia in cancer: a systematic review of the literature. Am J Med 2004;116 Suppl 7A:11S-26S.
20. Dunst J, Kuhnt T, Strauss HG, Krause U, Pelz T, Koelbl $\mathrm{H}$, et al. Anemia in cervical cancers: impact on survival, patterns of relapse, and association with hypoxia and angiogenesis. Int J Radiat Oncol Biol Phys 2003;56:77887.

21. Lim S, Lee CM, Park JM, Jung SY, Lee KB. An association between preoperative anemia and poor prognostic factors and decreased survival in early stage cervical cancer patients. Obstet Gynecol Sci 2014;57:471-7.

22. Rades D, Schild SE. Challenges of anemia correction during radiotherapy and chemoradiation. Eur Med Oncol 2012;5:35-8.

23. Koulis TA, Kornaga EN, Banerjee R, Phan $T$, Ghatage $P$, Magliocco AM, et al. Anemia, leukocytosis and thrombocytosis as prognostic factors in patients with cervical cancer treated with radical chemoradiotherapy: a retrospective cohort study. Clin Transl Radiat Oncol 2017;4:51-56.

24. Bishop AJ, Allen PK, Klopp AH, Meyer LA, Eifel PJ. Relationship between low hemoglobin levels and outcomes after treatment with radiation or chemoradiation in patients with cervical cancer: has the impact of anemia been overstated? Int J Radiat Oncol Biol Phys 2015;91:196-205.

25. Cho O, Chun M, Chang SJ, Oh YT, Noh OK. Prognostic Value of Severe Lymphopenia During Pelvic Concurrent Chemoradiotherapy in Cervical Cancer. Anticancer Res 2016;36:3541-7.

26. Bogani G, Sabatucci I, Maltese G, Lecce F, Signorelli M, Martinelli $F$, et al. Chemotherapy-related leukopenia as a biomarker predicting survival outcomes in locally advanced cervical cancer. Eur J Obstet Gynecol Reprod Biol 2017; 208:41-5.

27. Ray-Coquard I, Cropet C, Van Glabbeke M, Sebban C, Le Cesne A, Judson I, et al. Lymphopenia as a prognostic factor for overall survival in advanced carcinomas, sarcomas, and lymphomas. Cancer Res 2009;69:5383-91.

28. Fyles A, Keane TJ, Barton M, Simm J. The effect of treatment duration in the local control of cervix cancer. Radiother Oncol 1992;25:273-9.

29. Girinsky T, Rey A, Roche B, Haie C, Gerbaulet A, Randrianarivello $\mathrm{H}$, et al. Overall treatment time in advanced cervical carcinomas: a critical parameter in treatment outcome. Int J Radiat Oncol Biol Phys 1993;27:1051-6.

30. Biewenga P, van der Velden J, Mol BW, Stalpers L, Schil- 


\section{Obstetrics \& Gynecology Science}

Feiya Shi, et al. CRT heme toxicities in cervical cancer

thuis MS, van der Steeg JW, et al. Prognostic model for survival in patients with early stage cervical cancer. Cancer 2011;117:768-76.

31. Onal C, Yildirim BA, Guler OC, Mertsoylu H. The utility of pretreatment and posttreatment lymphopenia in cervical squamous cell carcinoma patients treated with definitive chemoradiotherapy. Int I Gynecol Cancer 2018;28:1553-59.

32. Taguchi A, Furusawa A, Ito K, Nakajima Y, Shimizuguchi T, Hara K, et al. Postradiotherapy persistent lymphopenia as a poor prognostic factor in patients with cervical cancer receiving radiotherapy: a single-center, retrospective study. Int J Clin Oncol 2020;25:955-62.

33. Azab B, Camacho-Rivera M, Taioli E. Average values and racial differences of neutrophil lymphocyte ratio among a nationally representative sample of United States subjects. PLoS One 2014;9:e112361.

34. Warnke C, Dehmel T, Ramanujam R, Holmen C, Nordin $N$, Wolfram K, et al. Initial lymphocyte count and low BMI may affect fingolimod-induced lymphopenia. Neurology 2014;83:2153-7.

35. Borges TC, Gomes TL, Pichard C, Laviano A, Pimentel GD. High neutrophil to lymphocytes ratio is associated with sarcopenia risk in hospitalized cancer patients. Clin Nutr 2021;40:202-6.
36. Lee AY, Golden DW, Bazan JG, Kopec M, Pelizzari CA, Aggarwal $S$, et al. Hematologic nadirs during chemoradiation for anal cancer: temporal characterization and dosimetric predictors. Int J Radiat Oncol Biol Phys 2017;97:306-12.

37. Sini C, Fiorino C, Perna $L$, Noris Chiorda B, Deantoni $C L$, Bianchi $\mathrm{M}$, et al. Dose-volume effects for pelvic bone marrow in predicting hematological toxicity in prostate cancer radiotherapy with pelvic node irradiation. Radiother Oncol 2016;118:79-84.

38. Mell LK, Kochanski JD, Roeske JC, Haslam JJ, Mehta N, Yamada SD, et al. Dosimetric predictors of acute hematologic toxicity in cervical cancer patients treated with concurrent cisplatin and intensity-modulated pelvic radiotherapy. Int J Radiat Oncol Biol Phys 2006;66:1356-65.

39. Dyer BA, Zamarin D, Eskandar RN, Mayadev JM. Role of immunotherapy in the management of locally advanced and recurrent/metastatic cervical cancer. J Natl Compr Canc Netw 2019;17:91-7.

40. Ouh YT, Cho HW, Kim SM, Min KJ, Lee SH, Song JY, et al. Risk factors for type-specific persistence of high-risk human papillomavirus and residual/recurrent cervical intraepithelial neoplasia after surgical treatment. Obstet Gynecol Sci 2020;63:631-42. 